\title{
Intelligent Bionic Artificial Arms Control Based on sEMG
}

\author{
Shu Qiao*, Dan Ding and Ruiyou Zhu
}

Physical Testing Center Liaoning Shihua University, Fushun 113001, P.R. China

\begin{abstract}
The correlations between signal and muscle activities in different motion patterns can be achieved by analyzing and processing the surface electromyographical signal (sEMG). In this paper, the results obtained by pattern recognition have been used to command the virtual intelligent arm, established with Adams, to finish the designated actions. A control algorithm called Active Disturbance Rejection Control is adopted, which is independent of exact mathematical model of the controlled object and easy to carry out. It is able to guarantee the control precision under the condition of strong uncertain disturbance and strong unknown nonlinearity, due to which, it can be applied to the control of bionic arm with a large number of DOF.
\end{abstract}

Keywords: Surface electromyographical signal, Active Disturbance Rejection, Adams.

\section{INTRODUCTION}

Extract sEMG signals that correspond to the movement from the stump of the user; work out the motion pattern through analysis; drive the prosthetic to complete the motions correctly. This is the working principal of the intelligent bionic artificial arm. The intelligence of bionic arm is mainly embodied in that the bionic arm emulates the command from mind with the result of pattern recognition and control the bionic arm to complete the motion, by substituting the signal processing for the nerve impulse conduction process in the muscle.

The corresponding pattern recognition results can be achieved by analyzing and processing the sEMG signals. In this paper, we mainly employ the results as control signal to make the mechanical arm complete the corresponding motion correctly and stably. In order to improve the quality of life for the disabled, the dexterity of the bionic arm should be as comparable to the human hand as possible. In this paper, we adopt the virtual prototyping technology and design a bionic arm with 16 DOF by using Adams. Virtual Prototyping (VP) can not only establish three-dimensional body models and mechanical model of the system, but also analyze and evaluate the system performance, thereby providing parameter and basis for the design and manufacturing of the physical prototyping [1-3]. As one of the most popular virtual prototyping analysis software, Adams' technical maturity and easy to operate quality, is widely applied in the field of relevant mechanical product virtual prototype development. It has the advantage that it can help designer discover design flaws and propose improved approach during the product development stages through virtual experiment and test.

\footnotetext{
*Address correspondence to this author at the Physical Testing Center Liaoning Shihua University, Fushun 113001, P.R. China; Tel: 13704932290; Fax: 024-56866286; E-mail: 649041012@qq.com
}

A mathematical model was established based on the virtual bionic arm with Adams in this paper [4-6]. The Active Disturbance Rejection Controller (ADRC) was chosen to complete different movements. The ADRC, derived from PID controller, adopts the core concept of error feedback control. Conventional PID controller calculates an error value as the difference between a measured process variable and a desired set point. The error is used as control signal directly, causing the contradiction between responsiveness and overshoot. The ADRC, which consists of Tracking Differentiator (TD), Extended State Observer (ESO) and Nonlinear State error Feedback control law (NLSEF), can address to a certain degree the problems that exist in conventional PID controllers. The function of TD is to arrange transient course and give appropriate control signal, which can resolve the contradiction between rapidity and overshoot. ESO is used to solve the impact of uncertainties of the model and external perturbations. Different from general state observer, an extended state variable is designed in ESO to track the unknown part of the model and the external disturbance. ESO gives control signals to compensate for the total disturbance, which can ensure the zero steady-state offset without using integral feedback in PID. NLSEF is the combined error of all order derivatives of TD and the state variable of ESO. NLSEF and the compensation of ESO for the total disturbance together constitute the control variable. Nonlinear feedback is much better at minimizing the error between PV and SP than linear feedback is. Therefore, the weighted sum in PID is replaced by the nonlinear combination [7-9] in NLSEF.

This paper is organized as follows. Virtual arm designed with Adams and the kinetic models based on it are established in Section 2.1. The ADRC is briefly described in Section 2.2. The simulation results are presented in Section 3. The advantages of sEMG and the proposed technique are summarized in Section 4. 


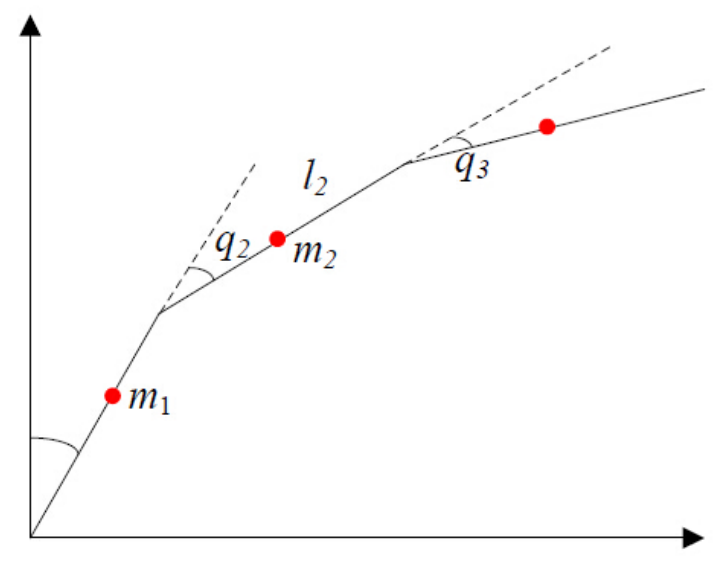

Fig. (1). Human Finger Model.

\section{CONTROL OF BIONIC ARM BASED ON SEMG SIGNAL}

\subsection{Dynamics Model of the Bionic Artificial Arm}

A model of bionic arm is established with the software Adams. Due to a lot of DOF, in this paper, we mainly focus on the three-dimensional model of complex human hand. A dynamics model is established based on the above model, and then ADRC technique is utilized to examine the validity and stability of the control. Simplify the human finger as three-connecting rod, as shown in Fig. (1).

$x_{1}=\left[\begin{array}{lll}q_{1} & q_{2} & q_{3}\end{array}\right]^{T}$,

Where $q_{1}, q_{2}, q_{3}$ denote three angles respectively.li, $m i, J i$ are for the length, mass, and the moment of the $i_{t h}$ connecting rod respectively. Assuming that the connecting rod is homogeneous and the barycenter is in the middle of the rod, the moment of inertia can be represented as

$J_{i}=\frac{1}{3} m_{i} l_{i}^{2}$

The red dots, $P_{1}, P_{2}, P_{3}$, are for the barycenter positions of the three knuckles, as shown in Fig. (1). The barycentric coordinates can be calculated by the following formulas:

$p_{1}=\left(\begin{array}{l}l_{1} \sin q_{1} \\ l_{1} \cos q_{1}\end{array}\right)$

$p_{2}=a a\left(\begin{array}{l}l_{1} \sin q_{1}+\frac{1}{2} l_{2} \sin \left(q_{1}+q_{2}\right) \\ l_{1} \cos q_{1}+\frac{1}{2} l_{2} \cos \left(q_{1}+q_{2}\right)\end{array}\right)$

$p_{3}=$
$\left(\begin{array}{l}l_{1} \sin q_{1}+\frac{1}{2} l_{2} \sin \left(q_{1}+q_{2}\right)+\frac{1}{2} l_{3} \sin \left(q_{1}+q_{2}+q_{3}\right) \\ l_{1} \cos q_{1}+\frac{1}{2} l_{2} \cos \left(q_{1}+q_{2}\right)+\frac{1}{2} l_{3} \cos \left(q_{1}+q_{2}+q_{3}\right)\end{array}\right)$

The corresponding velocity vector can be expressed as: $v_{i}=d p_{i} /_{d t}, i=1,2,3$. Let $x_{1}=\left[\begin{array}{lll}q_{1} & q_{2} & q_{3}\end{array}\right]^{T}, x_{2}=\dot{x_{1}}=$ $\left[\begin{array}{lll}\dot{q}_{1} & \dot{q}_{2} & \dot{q}_{3}\end{array}\right]^{T}$, then the kinetic-energy of system is:
$T\left(x_{1}, x_{2}\right)=\frac{1}{2} \sum_{i=1}^{3} m_{i} v_{i}^{2}$

According to Lagrange equation

$\frac{d}{d t} \frac{\partial}{\partial x_{2}} T\left(x_{1}, x_{2}\right)-\frac{\partial}{\partial x_{1}} T\left(x_{1}, x_{2}\right)=u$ lows:

The dynamic model of the finger can be described as fol-

$\left\{\begin{array}{c}\dot{x_{1}}=x_{2} \\ M\left(x_{1}\right) \dot{x_{2}}+C\left(x_{1}, x_{2}\right)=u\end{array}\right.$

Where

$u=\left[\begin{array}{lll}\tau_{1} & \tau_{2} & \tau_{3}\end{array}\right]^{\mathrm{T}}$, denote moment of force on each knuckle. ( 1 ), called inertial matrix, which is of symmetry and positive definiteness, and can be expressed as follows:

$M=\left[\begin{array}{lll}M_{11} & M_{12} & M_{13} \\ M_{21} & M_{22} & M_{23} \\ M_{31} & M_{32} & M_{33}\end{array}\right]$

Where

$$
\begin{aligned}
& M_{11}=\theta_{1}+\theta_{2}+\theta_{4}+2 \theta_{3} \cos q_{2}+2 \theta_{5} \cos \left(q_{2}+q_{3}\right) \\
&+2 \theta_{6} \cos q_{3} \\
& M_{12}=M_{21}=\theta_{2}+\theta_{4}+\theta_{3} \cos q_{2}+\cos \left(q_{2}+q_{3}\right) \\
&+2 \theta_{6} \cos q_{3} \\
& M_{13}=M_{31}=\theta_{4}+\theta_{5} \cos \left(q_{2}+q_{3}\right)+\theta_{6} \cos q_{3} \\
& M_{22}=\theta_{2}+\theta_{4}+2 \theta_{6} \cos q_{3} \\
& M_{23}=M_{32}=\theta_{4}+2 \theta_{6} \cos q_{3} \\
& M_{33}=\theta_{4}
\end{aligned}
$$

$\left(x_{1}, 2\right)=\left[\begin{array}{lll}C_{1} & C_{2} & C_{3}\end{array}\right]^{\mathrm{T}}$, is the sum vector of inertial centrifugal moment and inertial Coriolis moment. $C 1, C 2, C 3$ can be calculated by the following formulas:

$$
\begin{aligned}
C_{1}=-\theta_{3}\left(2 \dot{q}_{1}\right. & \left.+\dot{q}_{2}\right) \dot{q}_{2} \sin q_{2}-\theta_{5}\left(2 \dot{q}_{1}+\dot{q}_{2}+\dot{q}_{3}\right)\left(\dot{q}_{2}\right. \\
& \left.+\dot{q}_{3}\right) \sin \left(q_{2}+q_{3}\right) \\
& -\theta_{6}\left(2 \dot{q}_{1}+\dot{q}_{2}+\dot{q}_{3}\right) \dot{q}_{3} \sin q_{3}
\end{aligned}
$$




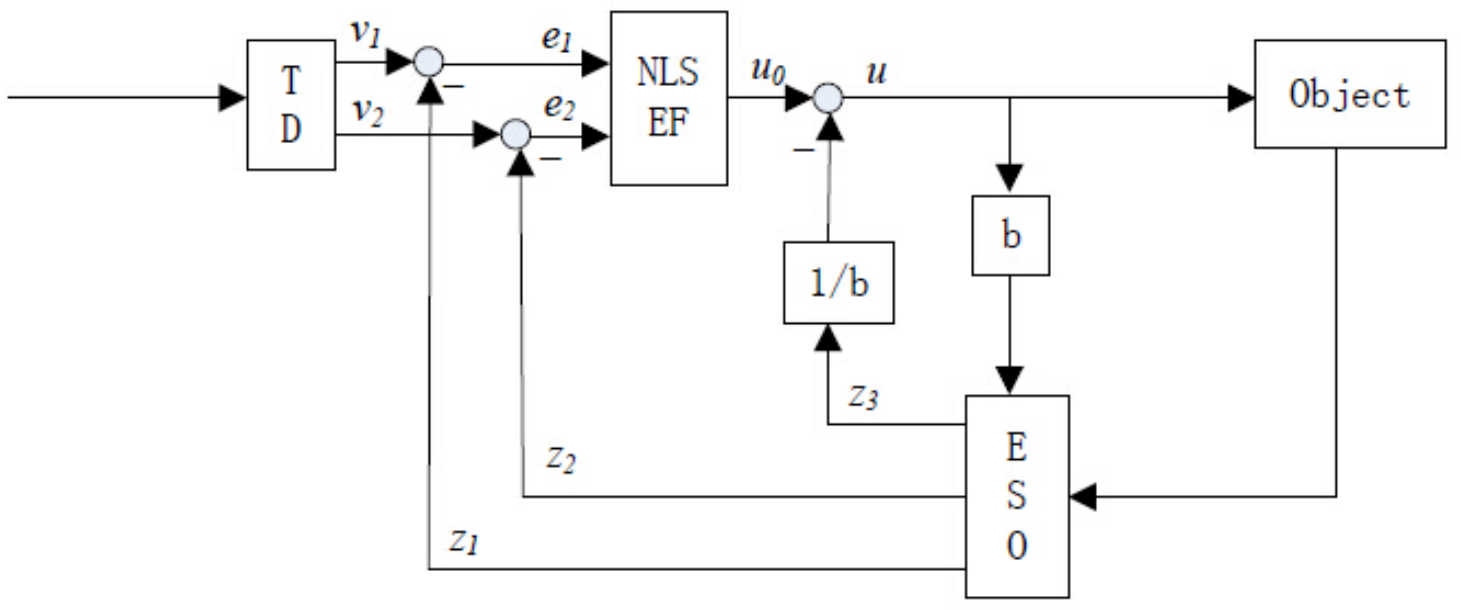

Fig. (2). Diagram of ADRC.

$C_{2}=\theta_{3} \dot{q}_{1}^{2} \sin q_{2}+\theta_{5} \dot{q}_{1}{ }^{2} \sin \left(q_{2}+q_{3}\right)-\theta_{6}\left(2 \dot{q}_{1}+\dot{q}_{2}+\right.$

$\left.\dot{q}_{3}\right) \dot{q}_{3} \sin q_{3}$

$C_{3}=\theta_{5} \dot{q}_{1}^{2} \sin \left(q_{2}+q_{3}\right)+\theta_{6}\left(\dot{q}_{1}+\dot{q}_{2}\right)^{2} \sin q_{3}$

where $\theta_{i}$ can be calculated by the following formulas:

$$
\begin{gathered}
\theta_{1}=J_{1}+\frac{1}{4} m_{1} l_{1}^{2}+\left(m_{2}+m_{3}\right) l_{1}^{2} \\
\theta_{2}=J_{2}+\frac{1}{4} m_{2} l_{2}{ }^{2}+m_{3} l_{2}^{2} \\
\theta_{3}=\left(\frac{1}{2} m_{2} l_{2}+m_{3} l_{2}\right) l_{3} \\
\theta_{4}=J_{3}+\frac{1}{4} m_{3} l_{3}{ }^{2} \\
\theta_{5}=\frac{1}{2} m_{3} l_{1} l_{3} \\
\theta_{6}=\frac{1}{2} m_{3} l_{2} l_{3}
\end{gathered}
$$

\subsection{Control of Bionic Artificial Arm Based on ADR Method}

Classic PID, which is widely applied in process control, adopts the control strategies that eliminate error caused due to the error between target and actual value $[10,11]$. However, PID has the following defects.

(1) Although the stability margin of PID is large, the margin of having good dynamic quality is small and the closed-loop dynamic quality is too sensitive to change the PID gain. Due to this, when the controlled object is in the environment that is constantly changing, the system needs to change PID gain according to the environmental change, which will restrict the practical application of PID.

(2) The essence of PID control is to eliminate error based on error feedback. However, it is not perfectly reasonable to use the error between target and a measured process variable directly, which makes the initial control force too large, thereby causing overshoot of system. This is the main reason that leads to the contradiction between responsiveness and overshoot in PID closed-loop control system.

(3) The feedback control signal is the weighted sum of proportional, integral, and derivative terms in PID. Whereas except for the situation that measures the rate of change of practical action, PI controllers are fairly common because there is no suitable differentiator, which restricts the control ability of PID.

(4) The proper combination of past error, present error and future error is used to produce control signal in PID. Classical PID adopts the weighted sum of them, compared with which, the nonlinear combination is more efficient.

Therefore ADRC method is introduced to control the dynamic model based on the bionic arm established above. Fig. (2) shows the diagram of ADRC.

$(t)$ in Fig. (2) represents reference input signal. Define $x_{3}=M^{(-1)}\left(x_{1}\right) C\left(x_{1}, x_{2}\right)$, which denotes the "total disturbances". Hence, the formula (7) can be extended as follows:

$\left\{\begin{array}{c}\dot{x}_{1}=x_{2} \\ \dot{x}_{2}=x_{3}+b u \\ \dot{x}_{3}=g(t)\end{array}\right.$

Where $b=M^{(-1)}\left(x_{1}\right)$

ADRC is composed of three parts: TD, ESO, and NLSEF. ESO, the core of ADRC, transforms the object model with the method of two-channel compensation, making the nonlinear indefinite system approximately linear and definite.

$$
\left\{\begin{array}{c}
\varepsilon=z_{1}(k)-x_{1}(k) \\
\dot{z}_{1}=z_{2}-\beta_{1} \varepsilon \\
\dot{z}_{2}=z_{3}-\beta_{2} \operatorname{fal}\left(\varepsilon, \alpha_{1}, \delta\right)+b u \\
\dot{z}_{3}=-\beta_{3} \operatorname{fal}\left(\varepsilon, \alpha_{2}, \delta\right)
\end{array}\right.
$$


Table 1. Parameters of Virtual Bionic Hand.

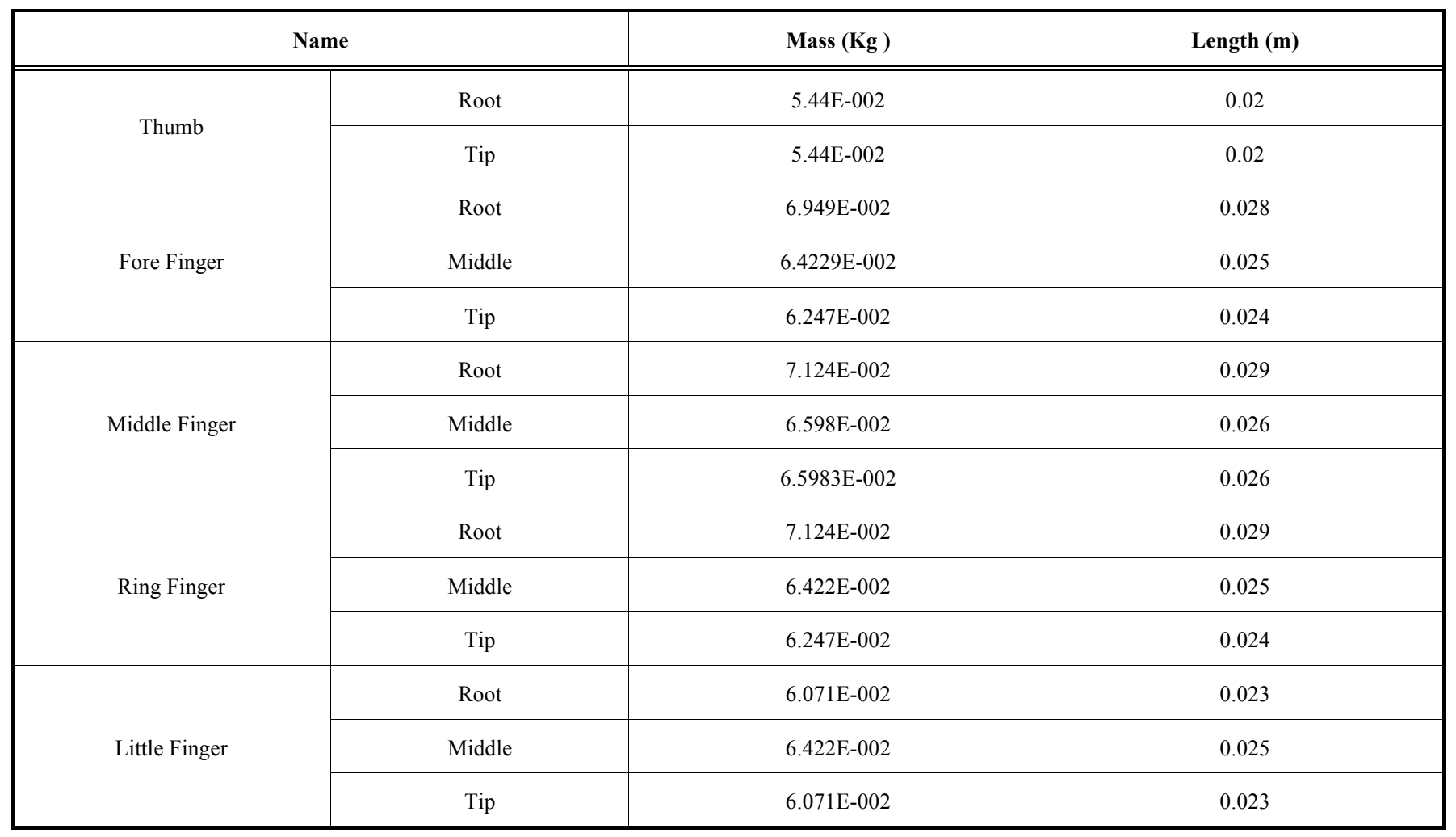

Where $\beta_{1}, \beta_{2}, \beta_{3}>0,1>\alpha_{1}, \alpha_{2}>0$, are design parameters. Function $\mathrm{fal}$ is as follows.

$\operatorname{fal}(\varepsilon, \alpha, \delta)=\left\{\begin{array}{c}|\varepsilon|^{\alpha} \operatorname{sign}(\varepsilon),|\varepsilon| \geq \delta \\ \frac{\epsilon}{\delta^{1-\alpha}},|\varepsilon| \leq \delta\end{array}\right.$

$\delta$ denotes the width of the linear interval. It is used to prevent the slope of the error curve being too large when approaching the origin, thereby eliminating the high frequency pulse. $\beta 1,2, \beta 3$, feedbacks of state error feedback, mainly influence the rate of convergence of ESO. The lager the parameter is, the smaller the latency on disturbance estimation is and the more quickly it converges. However, if the parameter is too large, the observer will oscillate, and the inhibition on the noise will be weakened.

Besides tracking reference input signal $(t)$ and arranging transition process, the main function of TD is to soften the change of $(t)$, to reduce the overshoot of system output and improve the robustness of ADRC. According to the secondorder steepest switching system, a second-order TD is expressed as follows.

$\left\{\begin{array}{c}\dot{v}_{1}=v_{2} \\ \dot{v}_{2}=-R \operatorname{sign}\left(v_{1}-v(t)+\frac{\left|v_{2}\right| v_{2}}{2 R}\right)\end{array}\right.$

In order to get rid of oscillation when approaching the origin, an appropriate linear region is added near the switch line. Replacing sign function $s i(\cdot)$ by linear saturation function, an efficient second-order TD can be obtained. It is expressed as follows. $\left\{\begin{array}{c}\dot{v}_{1}=v_{2} \\ \dot{v}_{2}=-\operatorname{Rsat}\left(v_{1}-v(t)+\frac{\left|v_{2}\right| v_{2}}{2 R}, \delta\right)\end{array}\right.$

where

$\operatorname{sat}(A, \delta)=\left\{\begin{array}{c}\operatorname{sign}(A),|A| \geq \delta \\ \frac{A}{\delta},|A| \leq \delta\end{array}\right.$

NLDEF is the nonlinear combination of all order derivatives of TD and the state variable estimation error of ESO. NLSEF and the compensation of ESO for the total disturbance together constitute the control variable. Modify it to linear interval and adopt function $f(t)$, in order to prevent the slope of the nonlinear curve being too large when approaching the origin, which can cause the problem of flutter in high frequency steady state.

$\left\{\begin{array}{c}e_{1}=v_{1}-z_{1} \\ e_{2}=v_{2}-z_{2} \\ u_{0}=k_{1} \cdot \operatorname{fal}\left(e_{1}, \alpha_{1}, \delta\right)+k_{2} \cdot \operatorname{fal}\left(e_{2}, \alpha_{2}, \delta\right) \\ u=u_{0}-\frac{z_{3}(k)}{b}\end{array}\right.$

\section{SIMULATION EXPERIMENT AND RESULTS ANALYSIS}

First, a simulation experiment with Matlab is conducted. In order to validate the control effect on the mechanical hand of ADR algorithm, the result of signal pattern recognition is chosen as the expected value. Parameters of the virtual bionic hand are shown in Table 1, according to the physiological characteristics of normal adult's right hand. 


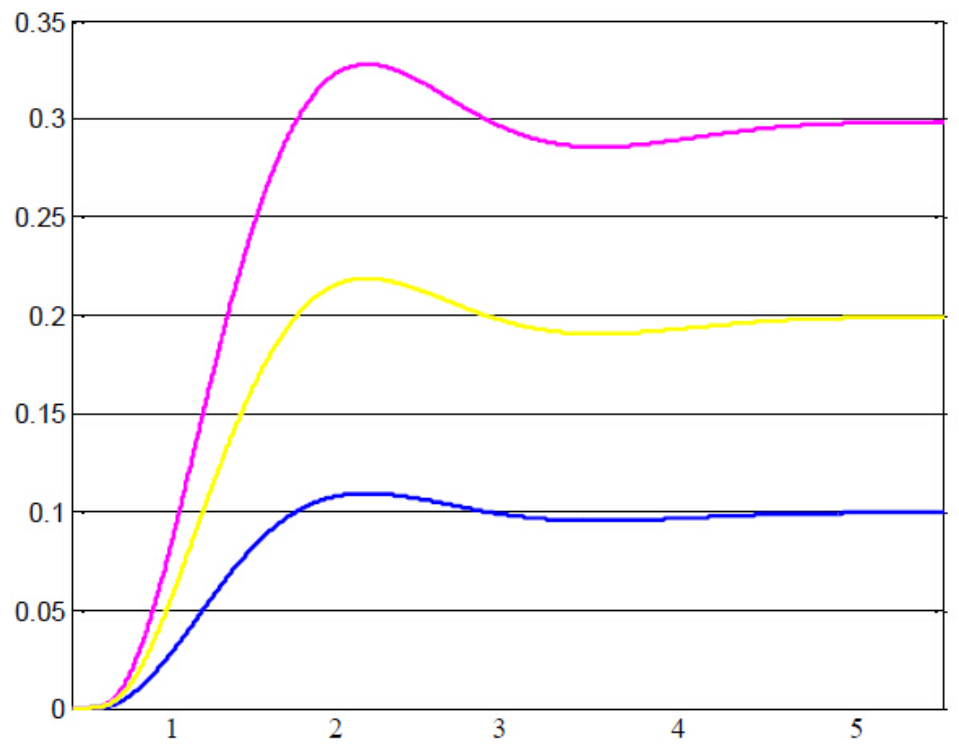

Fig. (3). Simulation Result of the Forefinger Control.

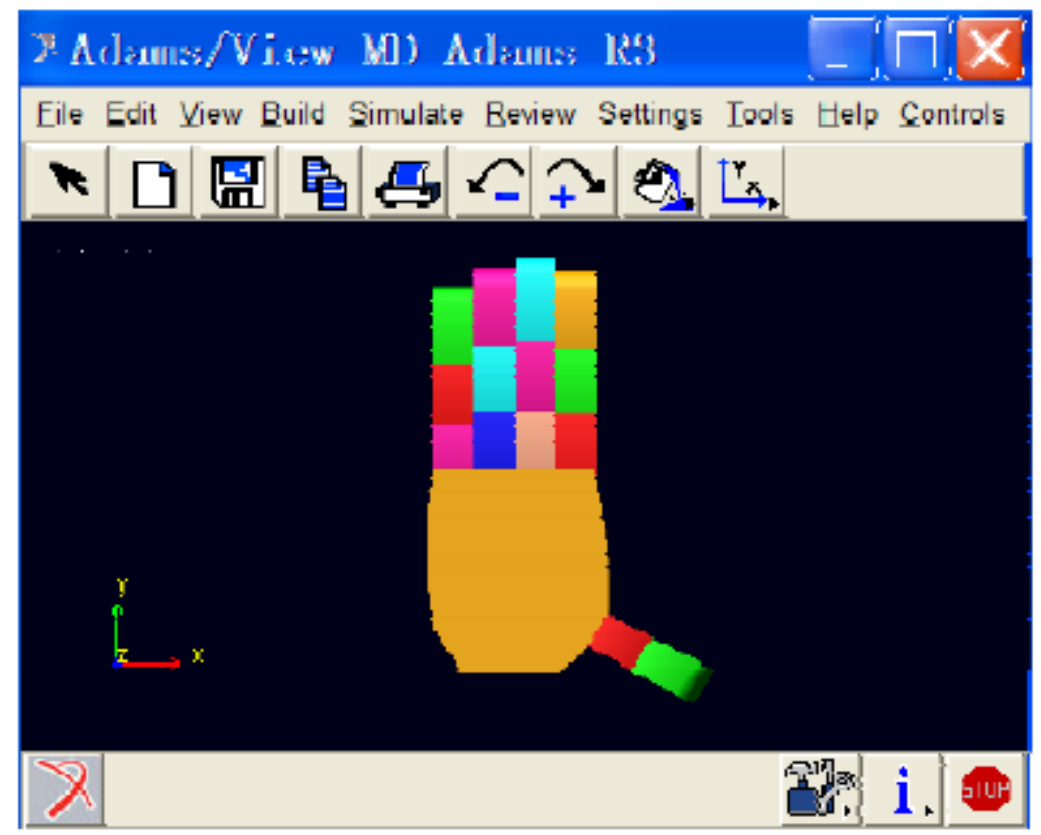

Fig. (4). Model of Bionic Hand with Multiple DOF designed by Adams.

Choose $\alpha_{1}=0.5, \alpha_{2}=0.25, k_{1}=5, k_{2}=1$ as the parameters of NLSEF; $\beta_{1}=\beta_{2}=\beta_{3}=500, \delta=0.01$ as the parameters of ESO. Establish the dynamic model of the hand, choose step response signal as system input, and verify the control effect of the algorithm. Take forefinger as an example, use $v=\left[\begin{array}{l}0.2 \\ 0.3 \\ 0.1\end{array}\right]$ as signal pattern recognition result; control each knuckle to track the desired signal $v$ from zero initial state. The control effect is shown as Fig. (3). The yellow curve is for fingertip motion, the purple for the middle and the blue for root.
Fig. (3) shows that the three knuckles of the forefinger can all reach the set value, which means the ADRC is able to command the finger to complete the specified movement.

Substitute the virtual bionic model designed with Adams for the dynamic model. The joint simulation of Matlab and Adams is conducted to validate the control effect on the virtual prototyping. The bionic arm with multiple DOF designed with Adams is shown as Fig. (4).

In order to improve the performance of virtual bionic hand and increase its flexibility, the artificial hand should be highly similar to real human hand. Its thumb has two 


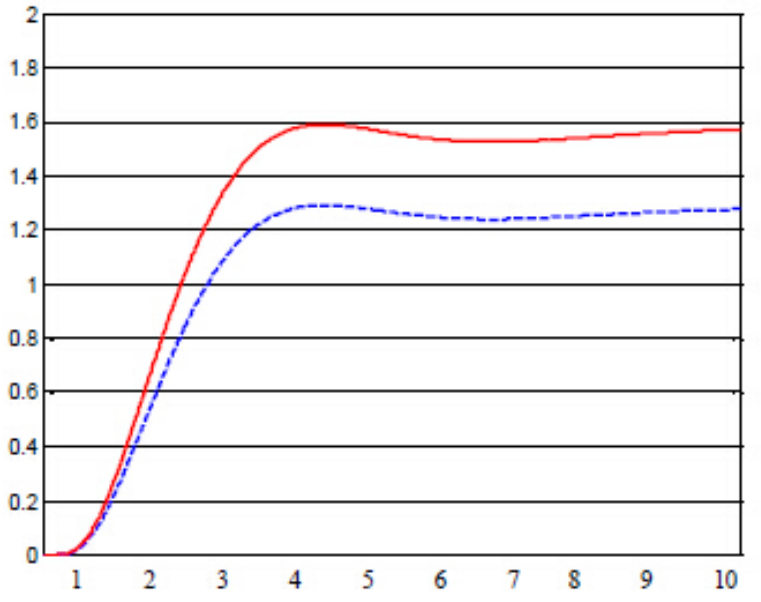

(a) Control Effect of Thumb Knuckles

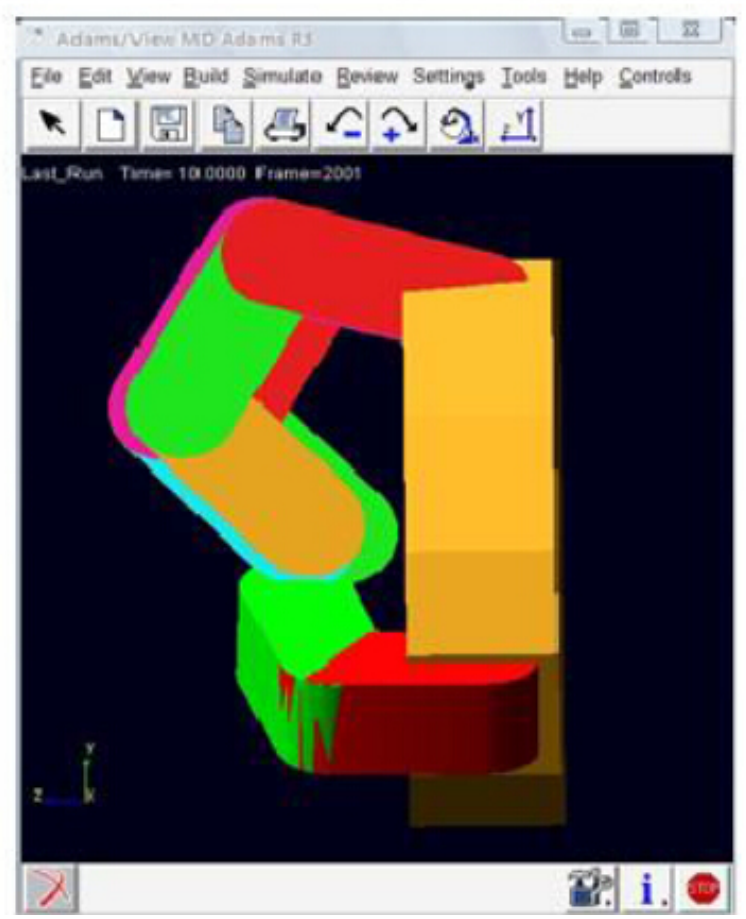

(c) Motion implementation in Adams (Side)

Fig. (5). Control effect of Fingers when Making Fist.

knuckles and each of the other four fingers has three knuckles. The root of thumb which has three DOF is relatively flexible. In order to control conveniently, let it have only one DOF and the angle between it and the other four fingers is about 120 degree when designed with Adams. By designing like this, the thumb with the other four fingers can help the user complete most hand motions in daily life, such as force grasping, precision grasping and gesture, through simple control.

Control the virtual bionic hand to make a fist. The root of thumb rotates 90 degrees, the tip rotates 75 degrees and the

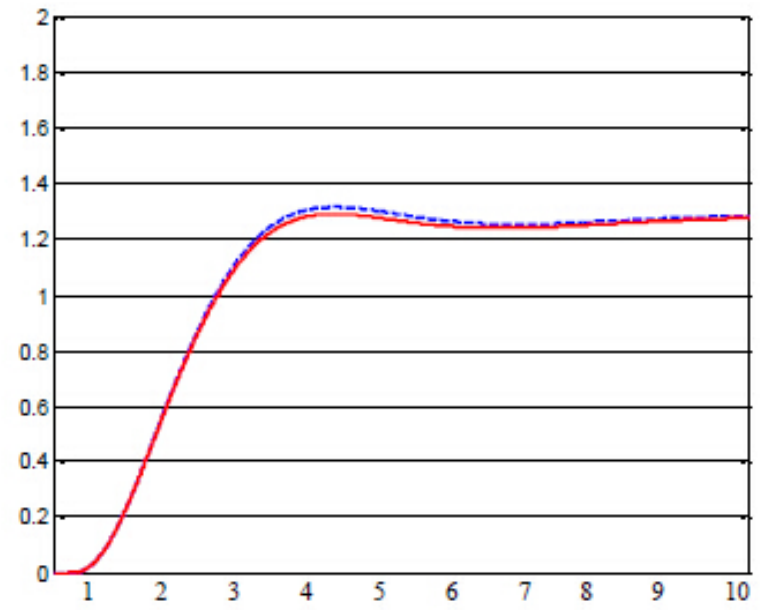

(b) Control Effect of Forefinger Knuckles

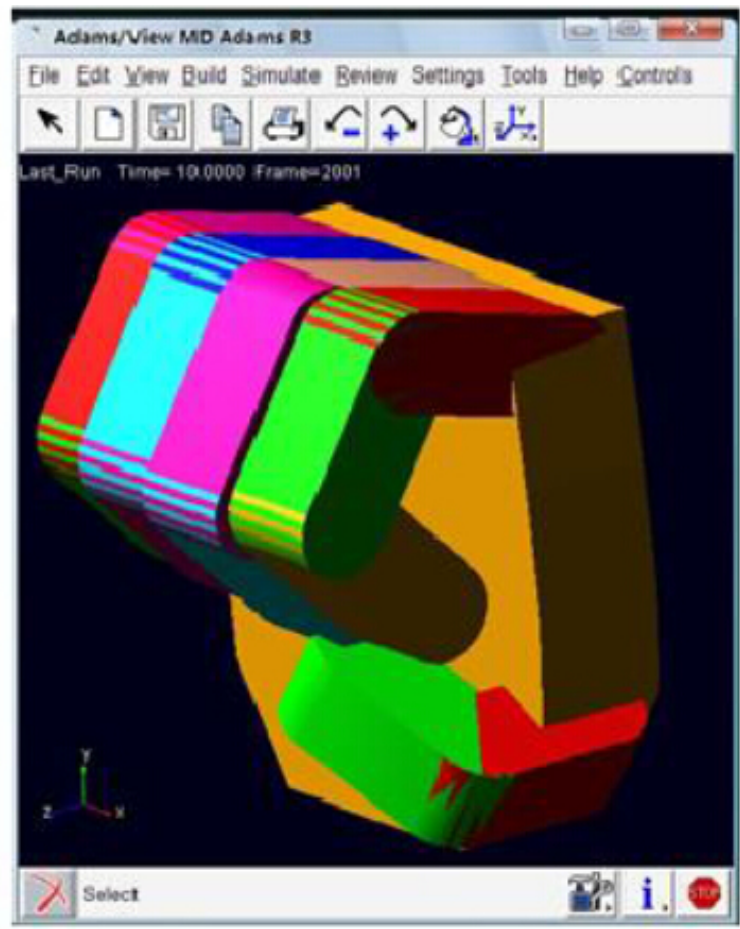

(d) Motion implementation in $\operatorname{Adams}\left(45^{\circ}\right)$

knuckles of the other four fingers rotate 75 degree when making a fist. Taking thumb and forefinger as an example, Fig. (5) depicts the control effect. The red curve in Fig. (5a) is for the root of the thumb and the blue is for the tip of the thumb. The red curve, blue curve and the green curve in Fig. (5b) represent control effect of the root, middle and tip of the forefinger respectively. Fig. (5c) and Fig. (5d) represent the simulation results in Adams from different angles. It's obvious that the bionic arm can finish making a correct fist.

Control the virtual arm to complete "victory" gesture. Root and tip of the thumb need to rotate 90 degree. Root of 


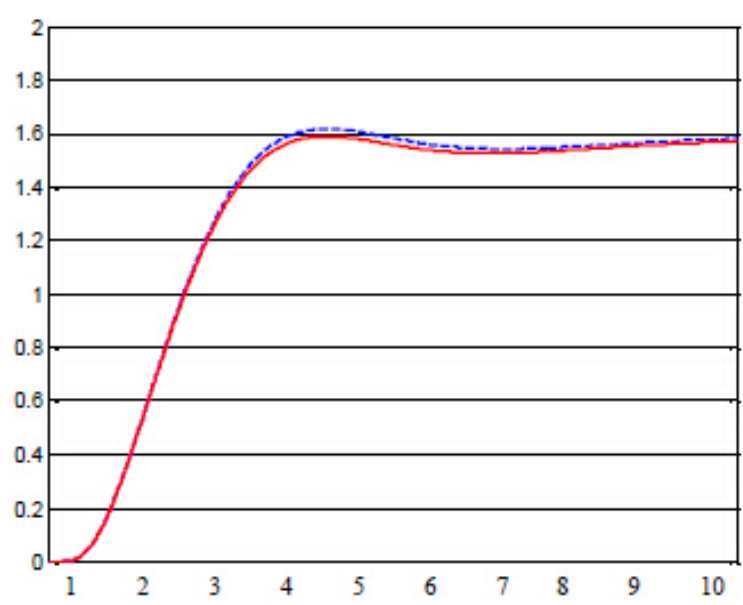

(a) Control Effect of Thumb Knuckles

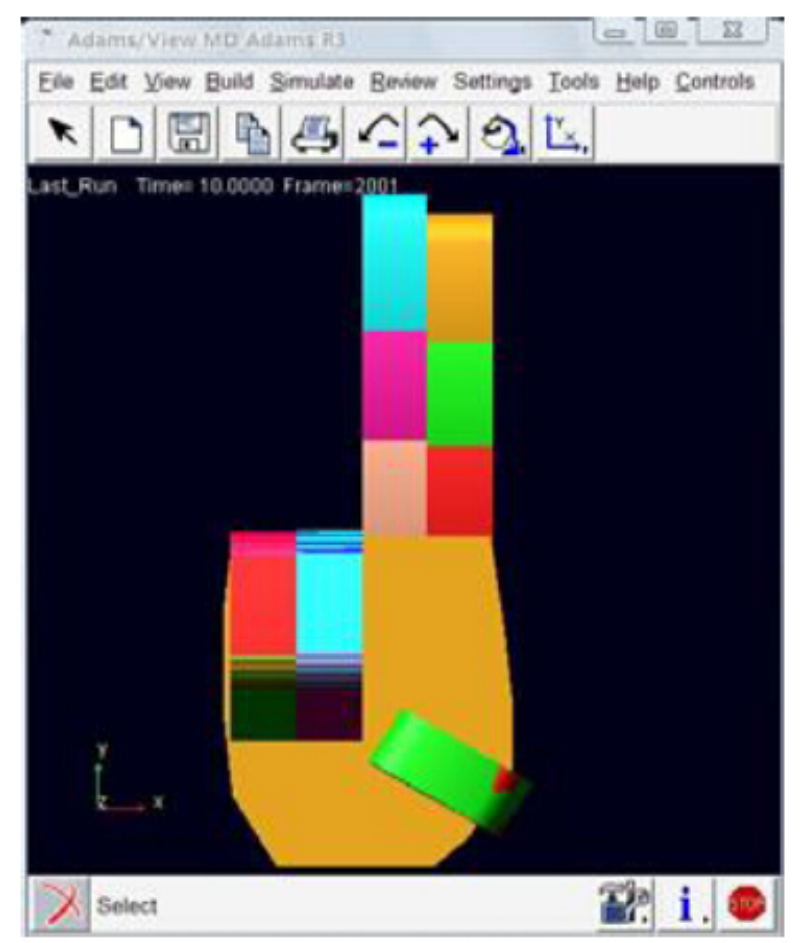

(c) Motion implementation in Adams (Front)

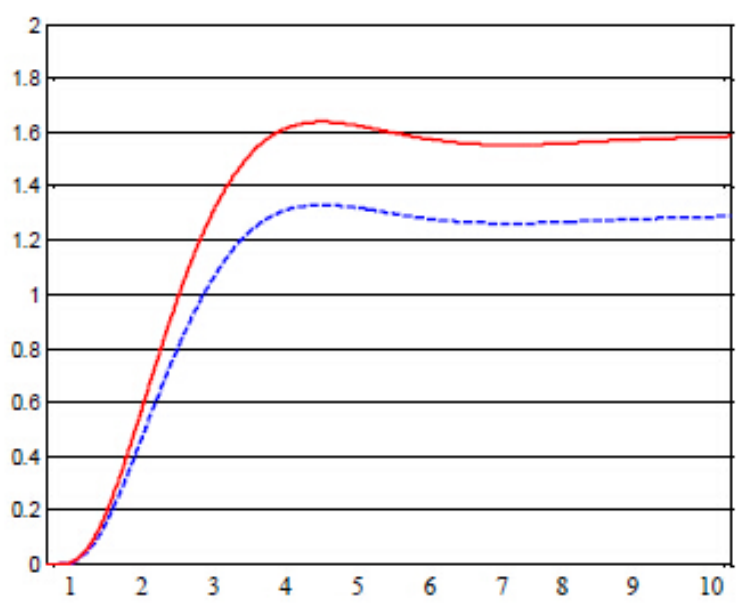

(b) Control Effect of Forefinger Knuckles

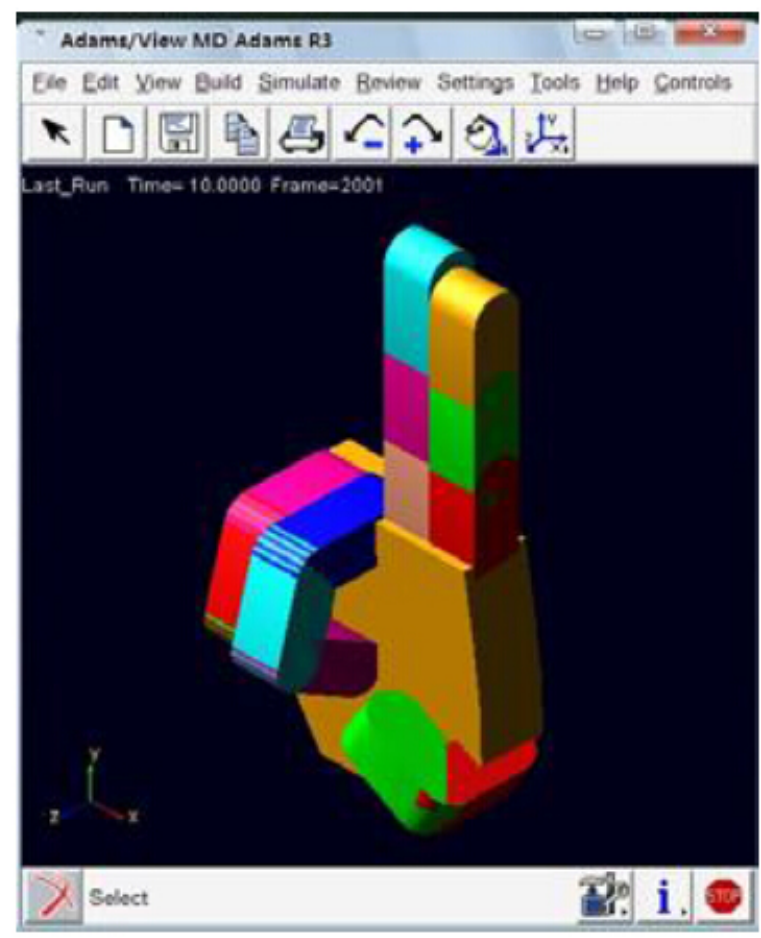

(d) Motion implementation in Adams (Top)

Fig. (6). Control effect of different fingers when making gesture "victory".

the ring finger and root of little finger rotate 90 degree, and middle and tip of them rotate 75 degree. Taking thumb and ring finger as an example, the control effect is depicted in Fig. (6). The red curve in Fig. (6a) is for the root of the thumb and the blue is for the tip of the thumb. The red curve, blue curve and the purple curve in Fig. (6b) represent control effect of the root, middle and tip of the ring finger respectively. Fig. (6c) and Fig. (5d) represent the simulation results in Adams from different angles. It's obvious that the virtual hand can finish the specified action correctly.
We can draw the following conclusion from the joint simulation result, as shown in Fig. (5) and Fig. (6). According to the angle of finger knuckles alone, ADRC gets angular velocity from ESO. ADRC considers the coupling relation among knuckles and the whole uncertainties act on the controlled object as unknown disturbance to make compensation for the system performance. NLSEF algorithm is employed instead of PID algorithm and the controller can command the finger to reach the specified angle correctly and steadily by setting suitable parameters. 


\section{CONCLUSION}

The sEMG, which is of incomparable superiority, has become a research focus in the field of intelligent bionic. It is safe and can be obtained easily, using the processing result of which as control input can ensure the accuracy and flexibility of the prosthetic, and decrease product cost at the same time. In this paper, Adams is utilized to design artificial arm with $16 \mathrm{DOF}$, the mathematical model of which is established by using kinematics and dynamics analysis. ADRC is introduced into bionic artificial arm. It is simple and independent of exact mathematical model of the controlled object. ADRC sees all the uncertainties as internal disturbances. It gives compensation by estimating the input and output of the model. This method has small or even no overshoot, which can control the virtual bionic arm to complete the specified movements correctly. The simulation result proves that it is feasible to use sEMG as control signal.

\section{CONFLICT OF INTEREST}

The authors confirm that this article content has no conflicts of interest.

\section{ACKNOWLEDGEMENTS}

This work was supported partially by the National Natural Science Foundation of People's Republic of China (61301300 and 61302058).

\section{REFERENCES}

[1] G.Q. Wang, Virtue prototyping technology and its application on Adams, M. Northwestern Polytechnic University Press, Xian, 2002.

[2] H.L. Wang, L.P. Zhao, and F.M. Chen, Application of mechanical design software, M. Xian JiaoTong University Press, Xian, 2007.

[3] W.D. Wei. Virtue prototyping technology and examples of its application in Adams, M. BeiHang University Press, Beijing, 2008.

[4] J.Q. Han, Nonlinear law of state error feedback-NLSEF, J, Control and Decision, vol. 10, no. 3, pp. 221-2231, 1995.

[5] J.Q. Han. From PID to ADRC technology, J. Control Engineering, vol. 9, no. 3, pp. 13-18, 2002.

[6] J.Q. Han, and R. Zhang. Error analysis of second-order ESO, J. Journal of System Science and Mathematical Science, vol. 19, no. 4, pp. 465-471, 1999.

[7] Y. Hang, and J.Q. Han, Design and analysis of nonlinear continuous second-order ESO [J], Science Bulletin, vol. 45, no. 13, pp. 1373-1379, 2000

[8] H.S. Li, and X.F. Zhu, Research on parameter tuning and optimization of ADRC, Journal of Control Engineering, vol. 11, no. 5, pp. 419-423, 2004

[9] J.Q. Han, ADRC technology -compensating for uncertainties by estimation [M], National Defense Industry Press, Beijing, 2009.

[10] W.Q. Pan, H.R. Xiao, and C. S. Wang, Design of Ship Course Controller Based on Optimal Active Disturbance Rejection Technique, C. International Conference on Intelligent System Design and Engineering Application, Shandong, China, pp. 582-585, 2010

[11] Y.J. Li, Y. F. Lu, and C. Hu. An Active Disturbance Rejection Control Technique for the Turntable System with Motor Moment Fluctuation, C.8th IEEE International Conference on Control and Automation, Shenzhen, China, pp. 80-85, 2010.

(C) Qiao et al.; Licensee Bentham Open.

This is an open access article licensed under the terms of the Creative Commons Attribution Non-Commercial License (http://creativecommons.org/licenses/by$\mathrm{nc} / 4.0 /$ ) which permits unrestricted, non-commercial use, distribution and reproduction in any medium, provided the work is properly cited. 